\title{
Bibliothekspädagogik und die Arbeit der AG Benutzerschulung des Landesverbandes Thüringen im DBV ${ }^{1}$
}

Vortrag am 20.09.2005 in Würzburg auf dem Fortbildungstag des Landesverbandes Bayern im VDB

Überarbeitete Fassung vom 27.09.2005

Holger Schultka

Inhalt:

1. Warum „Bibliothekspädagogik“?

2. Kompetenzen entwickeln - pädagogische Arbeit (nicht nur) in Bibliotheken

3. Bibliothekspädagogik, was ist das?

4. Bibliothekspädagogik in Hochschulbibliotheken

5. Die Arbeit der AG Benutzerschulung des Landesverbandes Thüringen im DBV

6. Ausblick

\section{Warum „Bibliothekspädagogik“?}

Hochschulbibliotheken haben die Entwicklung von Informationskompetenz als Schwerpunkt für ihre edukative Arbeit gewählt. Das Fokussieren auf die Informationskompetenz kann dazu führen, dass die anderen Kompetenzen aus dem Ensemble der Schlüsselqualifikationen aus dem Blick geraten. Hochschulbibliotheken versuchen gegenwärtig, in das Angebot des primären Bildungsträgers Hochschule vorzudringen und Anteile der Hochschullehre zu übernehmen bzw. mit dem Inhalt „Informationskompetenz“ neu zu etablieren. Hochschulbibliotheken präferieren dabei das formelle Lernen. Informelle Lernangebote werden nicht oder kaum unterbreitet.

Öffentliche Bibliotheken hingegen engagieren sich nicht nur in Sachen Informationskompetenz. Sie versuchen weitere Kompetenzen zu stärken, z. B. Lese-, Schreib-, Medien-, Lern-, Sprach- und interkulturelle Kompetenz. Öffentliche Bibliotheken realisieren Angebote des formellen und des informellen Lernens gleichermaßen.

Regional- und Landesbibliotheken beteiligen sich ebenso an der Entwicklung von Informationskompetenz, unterbreiten darüber hinaus aber regional-, kultur- und buchgeschichtliche Lernangebote. Überhaupt können Bibliotheken mit historischen Buchbeständen und anderen Spezialsammlungen (insbesondere die Forschungsbibliotheken) spezielle Lernangebote unterbreiten, die mit der Entwicklung von Informationskompetenz nichts mehr zu tun haben, sondern spezielle fachliche Lernangebote darstellen. Forschungsbibliotheken werden den Kulturtouristen zukünftig mehr Lernangebote unterbreiten müssen.

Die „Informationskompetenz“ ist somit nur ein Lernbereich aus einer Fülle von möglichen Lernbereichen.

Der Begriff „Bibliothekspädagogik“ ist geeignet, um alle edukativen Aktivitäten von Bibliotheken als pädagogische zu beschreiben sowie von der Fokussierung auf die Informationskompetenz wegzukommen und den Blick auf weitere Lerninhalte zu lenken.

\footnotetext{
${ }^{1}$ Vgl. auch: [1]: Schultka, Holger: Bibliothekspädagogik und die Arbeit der AG Benutzerschulung des Landesverbandes Thüringen im DBV. - In: Bibliothek : Forschung und Praxis. 29 (2005), Nr. 1, S. 59-65. [2]: Schultka, Holger: Bibliothekspädagogik. 2005 [Compuskript. Aufsatz erscheint voraussichtlich in: Bibliotheksdienst. 39 (2005), H. 11]
} 


\section{Kompetenzen entwickeln - pädagogische Arbeit (nicht nur) in Bibliotheken}

Das Thema des Fortbildungstages des Landesverbandes Bayern im VDB lautet: „Informationskompetenz stärken - Schlüsselqualifikationen lehren“. Interessant an dieser Themenstellung ist, dass die Informationskompetenz mit der ersten Aussage, „Informationskompetenz stärken“, zwar herausgestellt, einzeln genannt wird, doch mit der zweiten Aussage, „Schlüsselqualifikationen lehren“, wieder ins Ensemble der Schlüsselqualifikationen zurückverortet wird. Auch im Thema des Fortbildungstages ist die Öffnung hin zu weiteren Lerninhalten neben der Informationskompetenz enthalten.

Pädagogisches Handeln beginnt damit, dass man sich überlegt, was für einen Menschen man bilden, formen, erziehen will, was mit einer hohen Verantwortung verbunden ist. Insofern ist pädagogisches Handeln visionäres Handeln. Das Thema des heutigen Tages erzählt von diesem Ziel: Wir wollen, dass Menschen durch unser pädagogisches Handeln, informationskompetent werden bzw. ihre Informationskompetenzen weiterentwickeln. Wir wollen, dass Menschen mit Schlüsselqualifikationen ausgestattet werden.

Ein solch grundlegender Ansatz verlangt einen gesellschaftlichen Konsens, weil Bibliotheken nicht allein Menschen informationskompetent machen können. Bibliotheken sind sekundäre Bildungsträger. Die Idee der Entwicklung von Informationskompetenz muss von den primären Bildungsträgern (z. B. Schule, Hochschule) unbedingt mit getragen werden.

Pädagogische Entwürfe können zu gesellschaftlichen werden, z. B., wenn wir formulieren: Menschen müssen informationskompetent sein und über weitere Schlüsselqualifikationen verfügen, damit sie aktiv am gesellschaftlichen Leben teilnehmen können, die Gesellschaft kreativ mitgestalten, Berufschancen haben und einander achtend und verantwortlich begegnen. Solche oder ähnliche Formulierungen findet man auch in Texten zum lebenslangen Lernen oder in bildungspolitischen Veröffentlichungen, z. B. des Bundesministeriums für Bildung und Forschung ${ }^{2}$ oder der $\mathrm{EU}^{3}$.

Informationskompetente Menschen können selbstständig lernen, weil sie wissen, wo und wie

\footnotetext{
${ }^{2}$ Vgl.: Bundesministerium für Bildung und Forschung (Hrsg.): Ganztagsschule. Berlin : BMBF, 2005 (1stein : Spezial). - Vgl. dort insbesondere die Aussage von Andreas Schleicher, dem Leiter der Abteilung Bildungsindikatoren und Analysen bei der Organisation für wirtschaftliche Zusammenarbeit und Entwicklung (OECD) in Paris: „Unsere Bildungssysteme müssen diese Menschen [,Wissensarbeiter’] daher nicht nur mit solidem Fachwissen ausstatten, sondern in erster Linie mit der Fähigkeit und Motivation zu lebenslangem Lernen. Das setzt voraus, dass der Einzelne motiviert ist, ständig dazuzulernen, mit den erforderlichen kognitiven und sozialen Fähigkeiten ausgestattet ist, um eigenverantwortlich zu lernen, Zugang zu geeigneten Bildungsangeboten hat und schließlich entsprechende kulturelle Anreize findet. Daran, nicht an der Reproduktion von Fachwissen wird man den Erfolg zukünftiger Bildungsanstrengungen beurteilen.“ [Böhne, Sabine (Interv.) ; Schleicher, Andreas (Intervt.): Individuell fördern und Begabungen erkennen : Andreas Schleicher über Ganztagsschulen und ihre Bedeutung für die Zukunftsfähigkeit unserer Gesellschaft ; Interview. - In: Bundesministerium für Bildung und Forschung (Hrsg.): Ganztagsschule. Berling : BMBF, 2005 (1stein : Spezial), S. 26-27, dort auf S. 27] Wenn man diese Idee auf die Arbeit von Bibliotheken überträgt, so käme man zu der Aussage, dass sich Bibliotheken von Medien verwaltenden und Wissen speichernden Einrichtungen zu Kreativität fördernden und Lernen bewusst unterstützenden weiterentwickeln müssen.

${ }^{3}$ Vgl.: [1]: Europäische Union: Europäischer Raum des lebenslangen Lernens. [Online-Dokument] URL: http://europa.eu.int/scadplus/leg/de/cha/c11054.htm [Zugriff am: 19.09.2005]. [2]: Europäische Kommission: Ein europäischer Raum des lebenslangen Lernens / Generaldirektion für Bildung und Kultur ; Generaldirektion für Beschäftigung und Soziales. Luxemburg : Amt für amtliche Veröffentlichungen der Europäischen Gemeinschaften, 2002. - ISBN 92-894-3212-8. Zugl. online unter:

http://europe.eu.int/comm/dgs/education_culture/publ/pdf/lll/area_de.pdf. Vgl. dort z. B. S. 10: „[...] Ziele des [lebenslangen] Lernens [sind] z. B. Förderung aktiver und demokratischer Bürger, persönliche Entfaltung, soziale Eingliederung sowie Beschäftigungsaspekte. Außerdem baut lebenslanges Lernen auf bestimmten Grundsätzen auf, die auch für seine Umsetzung maßgeblich sind: die Lernenden im Zentrum,

Chancengleichheit sowie Qualität und Relevanz von Lernangeboten.“
} 
sie sich Informationen beschaffen können. Sie sind dazu in der Lage, veröffentlichtes Wissen zu prüfen und sich anzueignen und eigenes Wissen zu konstruieren.

An einem derart visionären pädagogischen Ziel kann - wie bereits gesagt - eine Bibliothek nicht allein arbeiten. An der Umsetzung der pädagogischen Vision müssen sich auf jeden Fall weitere Institutionen und Personen beteiligen, so z. B. die Hauptbildungsträger Kindergarten, Schule, Hochschule und Erwachsenenbildungseinrichtungen; natürlich auch die Eltern. Ebenso müssen sich die Kultus- und Wissenschaftsministerien engagieren. Sie müssen bildungspolitisch und -rechtlich handeln und den Bildungsrahmen mit schaffen. Ohne die Lernenden selbst, das sind für die Hochschulbibliotheken in erster Linie die Studierenden, geht natürlich gar nichts. Die Teilnehmer müssen erleben, dass die Hochschulbibliotheken - wenn sie Informationskompetenz und andere Schlüsselqualifikationen entwickeln, obwohl und trotzdem sie keine Hauptbildungsträger sind - die edukative Arbeit professionell leisten und das, was sie pädagogisch tun, den Teilnehmern nützlich ist.

Der Prozess, Informationskompetenz zu entwickeln, beginnt für einen Menschen nicht erst mit dem Besuch einer wissenschaftlichen Bibliothek bzw. einer Hochschule. Informationskompetent wird man bereits früher. Kinder müssen z. B. erlernen, wie man Fragen stellt. Schüler lernen, mit unterschiedlichen Nachschlagewerken umzugehen; sie lernen, die formale und die inhaltliche Struktur sowie die Funktion/den Zweck einer Quelle zu erkennen. Werkstattunterricht, entdeckendes und quellenbasiertes Lernen spielen dabei eine wichtige Rolle.

Hochschulbibliotheken werden von Menschen besucht, die bereits informationskompetent sind. Folglich stellt sich für den teaching librarian die Frage: Wie sollen sich die bereits Kompetenten weiterentwickeln? Welches Wissen und welche Fertigkeiten könnten ihnen wichtig sein? Was sollten sie lernen? Und was ist dabei überhaupt lernrelevant?

Auch in den „Information literacy competency standards for higher education“ der Association of College and Research Libraries wird auf die Notwendigkeit eines Bildungskonsenses hingewiesen. ${ }^{4}$ So hat z. B. die American Association for Higher Education die „Information literacy competency standards“ gebilligt, sodass die Standards als wesentliches Element der Hochschullehre angesehen werden können. Auch für die Schulausbildung sind Informationskompetenz-Standards formuliert worden, sodass sich die Idee der Entwicklung von Informationskompetenz sowohl durch die Schul- als auch durch die Hochschulausbildung zieht. In den „Information literacy competency standards for higher education“ wird darauf hingewiesen, dass Informationskompetenz die Basis für lebenslanges Lernen ist, egal welches Fach, welche Lernstufe und welche Lernumgebung sie betrifft. ${ }^{5}$

Will man am gesellschaftlichen Leben aktiv teilnehmen, muss man über eine Vielzahl von Qualifikationen verfügen. Man wird in der Lage sein müssen,

- zu lesen,

- zu schreiben,

- zu rechnen,

- zu kommunizieren,

- Informationen aufzufinden, zu sammeln, zu bewerten und zu verarbeiten,

- selbstständig zu lernen,

- kritisch zu denken,

- Wissen zu konstruieren,

\footnotetext{
${ }^{4}$ Vgl.: Association of College and Research Libraries: Information literacy competency standards for higher education : [January 2000]. [Online-Dokument] URL: http://www.ala.org/ala/acrl/acrlstandards/information literacycompetency.htm [Zugriff am: 15.09.2005]

${ }^{5}$ Vgl. ebenda
} 
- Wissen darzustellen (zu präsentieren).

- mit unterschiedlichen Menschen zusammenzuarbeiten,

- sich selbst zu erkennen und einzuschätzen.

Weitere Fertigkeiten lassen sich bestimmen.

Nutzt man zur Beschreibung den Kompetenz-Begriff, so lassen sich folgende Kompetenzen festlegen, die man als Schlüsselqualifikationen bezeichnen kann:

1. Lesekompetenz,

2. Medienkompetenz,

3. Informationskompetenz,

4. Schreibkompetenz,

5. Präsentationskompetenz,

6. Sozialkompetenz,

7. Selbstkompetenz,

8. Lernkompetenz,

9. Sprachkompetenz,

10. mathematisch-naturwissenschaftliche Kompetenz.

Dabei sollte man Fähigkeiten, Fertigkeiten und Kompetenzen klar voneinander unterscheiden. Fähigkeiten sind Leistungsdispositionen, um Handlungen ausführen zu können. Fertigkeiten sind verfestigte und trainierte Handlungsmuster. Kompetenzen sind Fertigkeitsbereiche, somit nie nur eine einzige Fertigkeit. Jemand, der z. B. lesekompetent ist, kann nicht nur Schrift erfassen, er kann auch Textsorten unterscheiden, die Hauptgedanken eines Textes erkennen, lineare und nicht lineare Texte lesen, im Text Unterstreichungen und Randbemerkungen anbringen, kursorisch lesen usw.

Die Frage ist nun, wie kann man Schlüsselqualifikationen, insbesondere Informationskompetenz, bei Menschen, die unterschiedliche Lernerfahrungen mitbringen, entwickeln und woran wird festgemacht, dass die Qualifikation entwickelt wurde. Damit sind wir bei pädagogischen Grundfragen angelangt:

a. Welchen Menschen wollen wir formen?

b. Wie, warum und wozu wollen wir das?

c. Wie können wir prüfen, dass wir unser Lehrziel erreicht haben? Wie kann der Lernende selbst prüfen, ob er das Lernziel erreicht hat?

d. Wie fördern wir die, die das Ziel nicht erreicht haben?

e. Welche didaktischen Situationen müssen wir schaffen, damit uns die Entwicklung von Menschen gelingt bzw. damit sich Menschen unter Ausnutzung ihrer eigenen Potenziale entfalten können? (Die didaktischen Situationen sind durch die neun Elemente wer lehrt wem was wann wo wie womit warum wozu gekennzeichnet; man könnte auch sagen: Lehrender, Lernender, Inhalt, Zeit, Ort, Methode, Hilfsmittel, Grund, Ziel.)

f. Die Gestaltung der didaktischen Situation und unser eigenes Verhalten als teaching librarians in ihr sind ganz stark von unserem Menschenbild und unserer Idee der Menschenbildung geprägt.

Zusammenfassend kann man sagen: Es gilt folgende Punkte zu bedenken, wenn man edukative Arbeit in Bibliotheken etablieren will:

1. Kompetenzen werden von den primären Bildungsträgern Elternhaus, Kindergarten, Schule, Hochschule, Erwachsenenbildungseinrichtungen entwickelt. Bibliotheken können sich an der Kompetenzentwicklung beteiligen, sind jedoch nie die alleinigen 
oder gar die ersten Kompetenzentwickler. Bibliotheken gehören zu den sekundären Bildungsträgern.

2. Menschen, die Bibliotheken aufsuchen, verfügen bereits über Qualifikationen. Insofern muss die Bibliothek den individuellen Entwicklungsstand der Lernenden respektieren, um bei den Lernenden Kompetenzen stärken zu können und um die Lernenden nicht durch Über- oder Unterforderung zu frustrieren.

3. Kompetenzentwicklung ist pädagogische Arbeit, d. h., Bibliotheken müssen, wenn sie bei Menschen Kompetenzen entwickeln wollen, pädagogische Denk- und Arbeitsweisen in die institutionalisierten bibliothekarischen Handlungsmuster integrieren.

4. Pädagogische Arbeit ist vielfältig, sie kann auf unterschiedliche Art realisiert werden. Dies unterscheidet die pädagogische Arbeit auch von der bibliothekarischen. Bibliothekarische Arbeit setzt auf Normierung. Pädagogische Arbeit hingegen benötigt die Varianz und befördert diese. Dass pädagogische Arbeit vielfältig ist, belegen Begriffe und Wörter wie z. B. lehren, unterrichten, Selbstlernmaterial, blended learning, Vortrag, Seminar, Führung, Diskussion, Fortbildung, formelles Lernen, informelles Lernen, Ausstellung, Freizeitpädagogik, e-learning, Museumspädagogik, Sozialpädagogik, Exkursion, Lernwerkstatt, Werkstatt-Unterricht, Vorlesung, quellenbasiertes Lernen und offene Arbeit.

5. Lehren und Lernen funktioniert nicht mechanistisch.

6. Kompetenzen können nicht durch reinen Faktenerwerb entwickelt werden. Sie entwickeln sich im und durch das Handeln. Wie man Probleme löst, lernt man, indem man Probleme löst. Recherchieren lernt man, indem man recherchiert. Schreiben lernt man, indem man schreibt. Und so weiter.

7. Kompetenzen sind stets kontextabhängig. Man kann sie zwar kontextunabhängig formulieren, doch besteht das Dilemma, wenn man kontextunabhängig beschreibt, darin, dass die Niveaustufe der Kompetenz unbeschrieben bleibt und von einer allgemeinen (beinahe abstrakten) Grundsituation ausgegangen wird, die es so natürlich nicht gibt, da jede Situation konkret ist. Gleichzeitig braucht man aber im pädagogischen Diskurs die modellierten Grundsituationen, um pädagogische Konzepte untereinander austauschen zu können. Man sollte sich bewusst sein, dass es eine Kluft zwischen Modell und Realität gibt. Man kann somit sagen, dass es die Kompetenz nicht gibt; es gibt Kompetenzen. Insofern wäre es auch günstiger, von Informationskompetenzen zu sprechen. Hinzu kommt, dass die Leistungsdispositionen von Menschen stets individuell, also verschieden sind.

8. Die Auffassungen davon, welche Kompetenzen entwickelt werden müssen, damit jemand am gesellschaftlichen Leben teilnehmen kann, haben sich im Laufe der Geschichte gewandelt; stets sind aber Könnens-/Fertigkeitsbereiche definiert worden. Warum betone ich dies? Teaching librarians sollten nicht in die „Alles-ist-neu-Falle“ tappen. Bibliotheken sind nicht die Ersten, die Kompetenzen definieren und entwickeln. Bibliotheken stellen sich derzeit, indem sie Kompetenzen, insbesondere Informationskompetenz, entwickeln, in ein bildungshistorisches Kontinuum.

Bibliotheken können sich neben den Hauptbildungsträgern (Elternhaus, Kindergarten, Schule, Hochschule, Erwachsenenbildungseinrichtungen) daran beteiligen, Schlüsselqualifikationen zu entwickeln. Bibliotheken können die Hauptbildungsträger bei der Entwicklung von Schlüsselqualifikationen unterstützen. Bibliotheken sollten nicht versuchen, die Hauptbildungsträger zu ersetzen oder mit diesen um die „Vorherrschaft“ im Bildungssektor konkurrieren. Zusammenarbeit ist notwendig! Bibliotheken haben die Chance im Rahmen ihrer Möglichkeiten (Personal, Finanzen, Arbeitsauftrag, Räume, Equipment usw.) - unter Ausnutzung ihrer lokalen Spezifik - pädagogische Arbeit als ein Serviceangebot zu entwickeln. 


\section{Bibliothekspädagogik, was ist das?}

\section{Bibliothekspädagogik ist Theorie und Praxis des pädagogischen Handelns in Bibliothe- ken.}

Erweiternd kann man definieren:

Definition 1: Bibliothekspädagogik ist ein Serviceangebot von Bibliotheken. Dieses ist darauf ausgerichtet, Kindern, Jugendlichen und Erwachsenen Lernangebote zu unterbreiten. Lernvorgänge sollen angeregt, unterstützt und aufrechterhalten werden. Die Lernangebote können sowohl formelle als auch informelle sein. Die formellen Lernangebote werden an den Anforderungen der Bildungsträger (z. B. Schule und Hochschule) oder an bildungspolitischen Forderungen ausgerichtet. Die informellen Lernangebote hingegen gehen von der Freiwilligkeit und Freiheit des Lernens aus; der Lernende bestimmt somit selbst, ob, wie und was er lernen will.

Definition 2: Bibliothekspädagogik ist die Theorie und Praxis des pädagogischen Handelns in Bibliotheken. Die Bibliothekspädagogik sucht nach Möglichkeiten, um Lernvorgänge bei den Bibliotheksbesuchern und -nutzern anzuregen und zu fördern. Die Bibliothekspädagogik versucht Lernangebote als Serviceangebote der Bibliothek zu etablieren und die Bibliothek als einen Lernort zu gestalten.

Definition 3: Bibliothekspädagogik ist visionäres, Demokratie förderndes Handeln. Sie unterstützt Menschen dabei, lesen und schreiben zu lernen, sich informieren zu können, Wissen zu konstruieren und Ideen zu entwickeln, um am gesellschaftlichen Leben aktiv teilnehmen zu können.

Wählt man beim Definieren einen ganz praktischen Ansatz, so müsste man formulieren: Bibliothekspädagogik ist das, was wir durch unsere Kreativität und unser Handeln in Bibliotheken an Bildungsideen umsetzen, ermöglichen, realisieren.

\section{Ziele einer Bibliothekspädagogik:}

Die Bibliothekspädagogik will einen Beitrag zur Kompetenzentwicklung bei Kindern, Jugendlichen und Erwachsenen leisten. Schlüsselqualifikationen sollen entwickelt werden. Insbesondere geht es um Informationskompetenz, Lesekompetenz, Schreibkompetenz, Präsentationskompetenz und Medienkompetenz. Darüber hinaus geht es um Sozialkompetenz, Selbstkompetenz und interkulturelle Kompetenz. Die Bibliothekspädagogik will Fertigkeiten trainieren und Methoden- und Faktenwissen vermitteln.

\section{Inhalte einer Bibliothekspädagogik:}

Als Inhalte einer Bibliothekspädagogik kommen die den geistigen Arbeitsprozess ausmachenden Inhalte in Frage: Recherchehilfsmittel, Nachschlagewerke, thematisches Recherchieren, inhaltliches Recherchieren, logische Operatoren, Trunkierungszeichen, Aufbau und Gliederung von Lexika und Bibliographien, Erstellen von Literaturverzeichnissen, Zitierregeln, Gliederung der Wissenschaften, Systematiken, das Alphabet, Medienarten, Arten von Veröffentlichungen usw. Darüber hinaus spielen folgende Inhalte eine Rolle: Kunst und Kultur, Streitfragen, Unterschied zwischen Meinung und Faktum, Buchgeschichte. Weitere Inhalte lassen sich bestimmen! Der Hauptinhalt wird sicherlich der Prozess des geistigen Arbeitens in Bibliotheken (also von der Fragestellung/vom Informationsbedürfnis bis hin zur fertigen Präsentation [meist schriftlichen Arbeit]) sein. 


\section{Realisationsweisen:}

Bibliothekspädagogik kann auf unterschiedliche Art umgesetzt werden. Die Lernveranstaltung ist nur eine Art der Realisation, wobei schon da unterschiedliche Veranstaltungsarten möglich sind, z. B. Vorlesung, Führung, Workshop, Training, Übung. Grob kann man folgende Realisationsweisen unterscheiden:

- Veranstaltungen (face to face),

- e-learning,

- blended learning,

- Didaktisierung der Internetseiten der Bibliothek,

- Ausstellungen oder Didaktisierung von Ausstellungen,

- Selbstlernmaterial,

- Gestaltung der Bibliothek oder von Teilen der Bibliothek als Lernraum/Lernatelier/Lernwerkstatt,

- $\quad$ betreute Bibliotheksbereiche für beratendes Lernen am point of use.

Sicherlich lassen sich noch weitere Formen bestimmen.

Arten der bibliothekspädagogischen Arbeit (Zielgruppen):

Bibliothekspädagogische Angebote können für unterschiedliche Zielgruppen entwickelt werden. Folgende Arten der bibliothekspädagogischen Arbeit können unterschieden werden:

1. pädagogische Arbeit mit Kindergartenkindern,

2. pädagogische Arbeit mit Schülern (Grundschülern, Haupt- und Regelschülern, Gymnasiasten),

3. pädagogische Arbeit mit Studierenden,

4. freizeitpädagogische Arbeit mit Kindern und Jugendlichen,

5. pädagogische Arbeit mit Familien,

6. Erwachsenenbildung,

7. Fortbildung (als spezielle Form der Erwachsenenbildung),

8. pädagogische Arbeit mit älteren und alten Menschen,

9. pädagogische Arbeit mit Soziogruppen, die spezielle Informationsbedürfnisse haben: $\mathbf{z}$. B. mit Migranten, Behinderten, Homosexuellen (separat und innerhalb der Bildungsbereiche 1 bis 8$)$.

Organisationsformen:

Die Bibliothekspädagogik kann organisatorisch fünf Wege beschreiten:

1. Die pädagogische Arbeit wird von der Bibliothek als Service entwickelt. Der Service wird auf die Zielgruppen abgestimmt. Eine Kooperation mit anderen Bildungsanbietern (insbesondere den Hauptbildungsträgern [z. B. Schule, Hochschule]) findet nicht statt. $=$ Vereinzelung

2. Die pädagogische Arbeit wird in Zusammenarbeit mit anderen Bildungsanbietern (insbesondere den Hauptbildungsträgern [z. B. Schule, Hochschule]) entwickelt. = Kooperation

3. Die pädagogische Arbeit wird für andere Bildungsanbieter (insbesondere für die Hauptbildungsträger [z. B. Schule, Hochschule]) geleistet. Der andere Bildungsanbieter erteilt also der Bibliothek den Auftrag und integriert die edukative Arbeit der Bibliothek in sein pädagogisches Angebot. = Integration 1 
4. Die Bibliothek integriert die pädagogische Arbeit anderer Bildungsanbieter in ihre Arbeit, z. B., indem sie Bildungsveranstaltungen anderer Anbieter zu sich einlädt. = Integration 2

5. Mischformen aus 1 bis 4 .

\section{Bibliothekspädagogik in Hochschulbibliotheken}

Wenn man in Hochschulbibliotheken bibliothekspädagogisch arbeiten will, wird man einige Entscheidungen bildungsorganisatorischer Art fällen müssen. Meines Erachtens gehören zu diesen:

- Wer soll sich an der edukativen Arbeit beiteiligen? Nur die Fachreferenten? Nur die Diplombibliothekare? Die Fachreferenten und die Diplombibliothekare?

- Wenn die Fachreferenten und die Diplombibliothekare edukative Arbeit leisten, wie ist die edukative Arbeit aufgeteilt? Übernehmen die Diplombibliothekare die fachübergreifenden Inhalte und die Fachreferenten die fachspezifischen?

- Sollen alle Fachreferenten und alle Diplombibliothekare bibliothekspädagogische Arbeit leisten oder wird diese Arbeit auf einen kleinen Kreis von Personen beschränkt, damit jeder dieser Gruppe ausreichend häufig Veranstaltungen durchführen kann, um genügend edukative Erfahrungen zu sammeln und pädagogische Fertigkeiten auszubilden?

- Wird die Gruppe angeleitet oder moderiert sich die Gruppe selbst?

- Werden Veranstaltungen durchgeführt oder sollen andere bibliothekspädagogische Realisationsweisen (z. B. Selbstlernmaterial) den Vorzug bekommen oder sollen Veranstaltungen und zugleich andere bibliothekspädagogische Realisationsweisen angeboten werden?

- Sollen einzelne Veranstaltungen oder ganze Veranstaltungsreihen angeboten werden?

- $\quad$ Sollen 45-minütige, 90-minütige, Vormittags-, Nachmittags-, Abend- oder Ganztagsveranstaltungen angeboten werden?

- Sollen in der Semesterpause und an Wochenenden Veranstaltungen angeboten werden?

- Sollen die Veranstaltungen auf Zuruf/Bestellung durchgeführt werden (somit stets auf die Bedürfnisse des Bestellers angepasst sein) oder sollen sie als zuvor geplante (normierte) Bausteine ${ }^{6}$ angeboten werden?

- Sollen die Veranstaltungen für die Studierenden der Hochschule fakultativ oder obligatorisch sein?

- Sollen die Veranstaltungen der Bibliothek in die Hochschullehre integriert, somit in die Studienordnungen/Curricula aufgenommen werden?

- Sollen die bibliothekspädagogischen Angebote ein separates Angebot der Bibliothek bleiben, in Kooperation mit der Hochschule entwickelt werden oder von der Bibliothek im Auftrage der Hochschule realisiert werden? Oder will die Bibliothek Bildungsangebote der Hochschule oder anderer Bildungsanbieter in die Bibliothek einladen?

- Will sich die Bibliothek als sekundärer Bildungsanbieter mit weiteren sekundären Bildungsanbietern, die auf dem Campus existieren (z. B. Rechenzentrum, Sprachenzentrum), zusammenschließen, um die sekundären edukativen Angebote für die Studierenden zu bündeln?

- Wie sollen sich die fachübergreifenden (basalen) Inhalte zu den sich darauf aufbauenden fachspezifischen (speziellen) Inhalten verhalten?

Lösungsmöglichkeit 1: Die basalen Inhalte werden zuerst und „fachunabhängig“ vermittelt. Anschließend werden die speziellen Inhalte fachgebun-

\footnotetext{
${ }^{6}$ Stichwort: Modularisierung
} 
den vermittelt.

Lösungsmöglichkeit 2: Die basalen und die speziellen Inhalte werden fachgebunden vermittelt. Diese Variante schafft den Nachteil, dass Personen, die mehrere Fächer studieren, in den verschiedenen Fächern mehrmals die Basics erfahren. Die Basics herauszuziehen und fachübergreifend und vorbereitend zu vermitteln, halte ich für die sinnvollere didaktische Entscheidung.

- Soll es auch pädagogische Angebote für Personen geben, die nicht zur Hochschule gehören?

- Welche Inhalte will die Hochschulbibliothek überhaupt vermitteln?

- Will die Bibliothek nur formelles Lernen fördern? Will sie auch informelles Lernen fördern? Welche Position nimmt sie zum informellen Lernen ein?

- Will die Bibliothek Hochschullehre übernehmen? Wenn ja, sollte die Bibliothek gemeinsam mit der Hochschule überlegen, wo die Hochschule ein Outsourcing von Hochschullehre hin zur Bibliothek vornehmen kann. Meines Erachtens ergeben sich da zwei Bereiche, das „Studium Fundamentale“ und das „Berufsfeld“. 7

Studium Fundamentale: „Das Studium Fundamentale zielt darauf, den Studierenden bestimmte Kompetenzen zu vermitteln, die im Rahmen des Fachstudiums nur in begrenzten[!] Rahmen erworben bzw. vermittelt werden können. Zu diesen Kompetenzen gehören insbesondere: Urteilskompetenz, Vermittlungskompetenz, ästhetisches Wahrnehmungsvermögen, soziale Kompetenz und interkulturelle Kompetenz. “8 Hier kann man die Informationskompetenz ergänzen!

Berufsfeld: „Der Studienbereich Berufsfeld bietet den Studierenden die Möglichkeit, Fähigkeiten und Fertigkeiten zu erwerben oder auszubauen, die im engeren Sinne auf bestimmte Berufsfelder vorbereiten.“9 „Es werden beispielsweise angeboten: [...] Einführungen in verschiedene Berufssparten (Verlags-, Schul-, Museums- und Bühnenwesen etc.).“ ${ }^{10}$ Veranstaltungen zur Berufssparte Bibliothekswesen wären möglich. Die Bibliothek kann darüber hinaus aber auch Lehrveranstaltungen zur Buchkunde, Handschriftenkunde, Restaurierung und zu anderen mit der Bibliothek im Zusammenhang stehenden Arbeits- und Themenfeldern durchführen. Diese Veranstaltungen könnten in den Studienrichtungen Geschichte, Kommunikationswissenschaft $u$. a. geeigneten Studienfächern angesiedelt sein. Um dies zu realisieren, ist es notwendig, dass die Hochschule die Bibliothek einlädt, Veranstaltungen zu diesen Themen durchzuführen. Die Hochschule müsste ein teilweises Outsourcing von Lehrhoheit in Richtung Bibliothek vornehmen.

Die Bibliothek sollte nicht versuchen, die Hochschullehre in den Haupt- und Nebenstudienrichtungen der Qualifikationen „Bachelor“ und „Master“ zu übernehmen. Um in den Haupt- und Nebenstudienrichtungen zu lehren, ist es erforderlich, dass man an die gegenwärtigen Lehr- und Forschungsdiskurse aktiv angebunden ist. Und gerade das ist die Bibliothek nicht. Sie ist zwar rezeptiv angebunden, was aber nicht ausreicht. Insofern sollte die Hochschule die propädeutischen Kurse in den Haupt- und Nebenstudienrichtungen selbst durchführen. Die Bibliothek kann auch hier nur bibliotheksbezügliche Inhalte übernehmen, was sie ja auch im „Studium Fundamentale“ und im „Berufsfeld“ tut. Die Bibliothek kann einen Beitrag zur Entwicklung von Informationskompetenz leisten und mit der Bibliothek im Zusammenhang stehende Inhalte

\footnotetext{
${ }^{7}$ Vgl. hierzu: Universität Erfurt: Prüfungs- und Studienordnung der Universität Erfurt für den BaccalaureusStudiengang in den Studienbereichen Studium Fundamentale und Berufsfeld. Fassung vom 24.10.2003. [OnlineDokument] URL: http://www.uni-erfurt.de/leitung/satzungsrecht/recht_ue/ba/doc/BA-PO-SF-BF-2_3_3_2.pdf [Zugriff am: 16.09.2005]- Amtliche Veröffentlichung am 29.10.2003. Registrier-Nr.: 2.3.3.2. Aktenzeichen: A0E09/107. Bearbeitungsstand: 10.10.2003

${ }^{8}$ A. a. O., S. 2; § 2, 1

${ }^{9}$ A. a. O., S. $6 ; \S 6,1$

${ }^{10}$ Ebenda; § 6, 2
} 
(z. B. Druckgeschichte, Provenienzforschung) vermitteln. Sie kann sich jedoch nicht in den Forschungsdiskurs der Hochschule und in die Lehrhoheit der Hochschule einmischen. Täte sie dies, würde sie einen Seitenwechsel vornehmen, eventuell sogar von ihren traditionellen Aufgaben abrücken. Bibliothekspädagogische Arbeit, verstanden als Seviceangebot, also als Ergänzung zu den Angeboten der Hauptbildungsträger, führt die Bibliothek nicht von ihren bisherigen Aufgaben weg, sondern stärkt die Bibliothek in ihrer Funktion als Kultur- und sekundäre Bildungseinrichtung.

Ob für größere bibliothekspädagogische Projekte, egal, ob es sich dabei um Veranstaltungen, um die Didaktisierung von Hompegages oder andere pädagogische Vorhaben handelt, auf Dauer Personalressourcen zur Verfügung stehen, muss jede Bibliothek im Einzelnen prüfen. Eine Möglichkeit, mit einer Vielzahl von Studierenden z. B. in einem Ein-Semester-Kurs umzugehen, ist blended learning (der Mix aus Präsenzanteil [face to face im realen und im virtuellen Lernraum], Selbstlernanteilen, Gruppentutorien und Konsultationen). - Natürlich könnte man auch nach einem behavioristischen Ansatz entscheiden, dass, da alle Studierenden bereits Erfahrungen mit dem Recherchieren und anderen Techniken des geistigen Arbeitens gesammelt haben, die Techniken nicht noch einmal unter Anleitung der Bibliothek beispielhaft geübt werden müssen, sondern es ausreicht, wenn die Studierenden zur Reflexion über ihre Erfahrungen angeregt werden und dann die gewonnenen Erkenntnisse in den nächsten Arbeitsprozess einbringen. Die Reflexionsphase könnte unter Anleitung in einer Einzelveranstaltung in der Bibliothek stattfinden, im betreuten Bereich am point of use in der Bibliothek passieren oder mit Selbstlernmaterial ausgelöst werden. Die nachfolgende Anwendungsphase könnte unter Anleitung in der Bibliothek stattfinden (als Einzelveranstaltung oder am point of use in Einzelbetreuung) oder von den Studierenden vollkommen selbstständig realisiert werden. Insofern ergibt sich die Frage: Muss die Bibliothek unbedingt ein Semester dauernde Veranstaltungen anbieten? Und wenn ja, warum will die Bibliothek überhaupt Semesterkurse durchführen?

Was könnte die Hochschulbibliothek noch in Sachen „Lernen“ tun?

\section{Medien-Aufkleber:}

Die Bibliothek könnte Aufkleber an bestimmten Medien anbringen, um diese dauerhaft zu kennzeichnen. Sie könnte z. B. studieneinführendes Material mit dem Aufkleber „Einführung“ ausstatten. Bücher, die die Hochschullehrer den Studierenden zur Lektüre empfehlen, könnten mit dem Aufkleber „Vom Hochschullehrer empfohlen“ versehen sein. Werke mit interdisziplinären Wissenschaftsstandpunkten könnten gekennzeichnet sein. Hier böten sich die Aufkleber „Frauenforschung“, „Queerstudies“, „Homostudies“, „Friedensforschung“, „Genderstudies“ an. Das Ziel der Aufkleber ist es, Lernanregungen zu geben, die Aufmerksamkeit der Studierenden zu lenken und informelles Lernen bei den Studierenden zu unterstützen.

\section{Die Gestaltung der Bibliothek oder Teile von ihr als Lernraum/Lernwerkstatt/Lernatelier:}

Hierzu möchte ich nur eine Idee entwickeln: Im Bibliotheksgebäude könnten z. B. an verschiedenen Stellen „Bildungswürfel“ oder „Bildungspfeiler“ aufgestellt werden. Vor diesen kann man stehen, an diesen kann man sitzen, man kann etwas herausnehmen, etwas anschalten, etwas anhören, ansehen, lesen usw. Die „Bildungswürfel“ bzw. „-pfeiler“ sind bestimmten Studienthemen gewidmet, z. B.: „Epochen der Kunstgeschichte“; „Deutsche Geschichte nach 1945“; „Bedeutende Werke der deutschen Literatur mit Interpretationen“; „Literatur und Literaturverfilmung“. Bei der Gestaltung der Würfel bzw. Pfeiler werden museumspädagogische Erfahrungen aufgegriffen. Es werden also nicht nur Medien wie in einem Semesterapparat zusammengestellt, sondern: Der „Bildungspfeiler“ „Epochen der Kunstgeschichte“ z. B. zeigt auf einem Zeitstrahl die Hauptepochen der Kunstgeschichte. Die Hauptwerke zu jeder 
Epoche sind als Abbildungen zu sehen, Hauptvertreter jeder Epoche werden mit Porträt gezeigt, Lebensdaten und Kurzlebenslauf sind zu erkennen. Man kann bestimmte Personen näher kennen lernen, indem man etwas über sie in einem Buch liest, das auf einem Regalbrett des „Bildungspfeilers“ steht. Man kann etwas erfahren, indem man eine CD anhört oder ein Video schaut. Per Mausklick erhält man eine Zusammenstellung wichtiger Werke aus der Bibliothek (Literaturliste); die Suchfrage wird erläutert. Mögliche Arbeitsthemen für Hausarbeiten werden formuliert. Die „Bildungspfeiler“ bzw. „-würfel“ besitzen einen hohen Aufforderungscharakter für informelles Lernen.

\section{Lerngewohnheiten respektieren:}

Bisher respektieren Hochschulbibliotheken kaum individuelle Lerngewohnheiten. Es muss zukünftig Stellen im Gebäude geben, wo man stehend, liegend oder sitzend lernen kann. Stehpulte und Liegematten wären notwendig. Es gibt Studierende, die, wenn sie ein Buch gesucht haben, gleich zwischen den Regalen sitzen bleiben wollen. Warum nicht tragbare, leicht abwaschbare Sitzkissen im Haus verteilen? Es gibt Mensch, die lieber allein lernen, andere wollen lieber in Gruppen lernen, einige brauchen leise Hintergrundmusik, andere wieder vollkommene Stille. Einzelarbeitsräume und Gruppenarbeitsräume sind notwendig. Menschen, die viel lernen, brauchen Bewegungs-/Kommunikations- und Ruhezonen in einem Gebäude, das meint, Stellen, wo man den Körper mal bewegen kann und richtig laut sprechen darf, und Stellen, wo man außerhalb der sonstigen Lernatmosphäre entspannen kann. Flächen, an denen das Auge angeregt wird, und Flächen, an denen das Auge entspannen kann, sollten im Gebäude vorhanden sein.

\section{Der behavioristische Ansatz:}

Obwohl ich bereits zuvor auf einen behavioristischen Ansatz Bezug genommen habe, möchte ich den vorherigen Gedanken nochmals vertiefen: Kompetenzen entwickelt man im Handeln. Fertigkeiten entstehen durch Handeln. Das heißt: Recherchieren lernt man, indem man recherchiert. Schreiben lernt man, indem man schreibt. Erfahrungen, die man beim Handeln sammelt, beeinflussen das zukünftige Handeln. Die Formel lautet: do - reflect - apply (tun reflektieren - anwenden). Wendet man dieses Modell auf die Zielgruppe der Studierenden an, könnte man z. B. zu drei bibliothekspädagogischen Überlegungen gelangen.

1. Die Recherche nach Literatur wird innerhalb unterschiedlicher Lehrveranstaltungen der Hochschule automatisch trainiert, weil die Studierenden Vorträge, Hausarbeiten usw. erarbeiten müssen. Die Bibliothek braucht deshalb keine Kurse anzubieten. Sie kann begleitende Selbstlernmaterialien einsetzen, die den Prozess „reflect“ bei den Studierenden unterstützen, sodass die Studierenden bei der nächsten Recherche die neuen Erkenntnisse in die Recherche einfließen lassen.

2. Die Recherche nach Literatur könnte beispielhaft in einer bibliothekspädagogischen Einzelveranstaltung simuliert werden. Die Veranstaltungsteilnehmer erhalten ein Thema oder wählen selbst ein solches. Zunächst recherchieren die Teilnehmer (= do). Dann folgt die Reflektionsphase und die Anwendungsphase. In der Anwendungsphase werden die in der Reflektionsphase gewonnenen Erkenntnisse eingebracht.

3. Die Bibliothek könnte eine Veranstaltung durchführen, in der auf die Phase „do“ verzichtet wird, da ja die Studierenden bereits während ihres Studiums Erfahrungen mit der Literaturrecherche gesammelt haben. Somit könnte man in der Veranstaltung gleich mit der Phase „reflect“ beginnen.

4. Die Bibliothek schafft betreute Recherchebereiche, in denen die Phasen „do“, „reflect“ und „apply“ vom Nutzer individuell durchlaufen werden und Bibliothekare individuelle 
Lernunterstützung ${ }^{11}$ geben.

\section{Unterrichtsveranstaltungen für Gymnasiasten:}

In Thüringen ist zu Beginn des Schuljahres 1999/2000 der Seminarfachunterricht in der gymnasialen Oberstufe eingeführt worden. Der Seminarfachunterricht erstreckt sich auf die Klassenstufen 10, 11 und die erste Hälfte von Klassenstufe 12. Schwerpunkt des Seminarfachunterrichts ist das Informationskompetenztraining. Im Unterricht wird eine vorwissenschaftliche Arbeit, die Seminarfacharbeit, erstellt. Diese muss schriftlich eingereicht und in einem Kolloquium verteidigt werden. Die Seminarfacharbeit stellt eine zusätzliche Prüfungsleistung für die Schüler dar. Hochschulbibliotheken können die Gymnasien bei der Gestaltung des Seminarfachunterrichts unterstützen.

\section{Weitere Möglichkeiten:}

Weitere Möglichkeiten, bibliothekspädagogisch in Hochschulbibliotheken zu arbeiten, sind z. B.:

quellenbasiertes Lernen,

- Ausstellungen,

- Bibliotheksbestände als Untersuchungsgegenstand promoten,

- Gestaltung von Bibliothekshomepages durch Studierende nach ihren eigenen Bedürfnissen (auf jeden Fall könnten die Internetlinks von den Studierenden selbst zusammengestellt werden),

- Didaktisierung der Bibliothekshomepage:

o zielgruppenspezifische Seiten für Studierende (diese unterteilt in: Anfänger und Fortgeschrittene), Forschende, Gymnasiasten usw.,

o mehr Übersichtstexte statt Fließtexte,

o mehr Visualisierungen,

o Lernpfade,

o Glossare,

o lotsende Elemente und Systeme.

\section{Die Arbeit der AG Benutzerschulung des Landesverbandes Thüringen im DBV}

Die Gründung der thüringenweiten AG Benutzerschulung ${ }^{13}$ geht auf den 7. Thüringer Bibliothekstag ${ }^{14}$, der am 27.10.2001 in Schmalkalden stattfand und dem Thema „Bibliotheken Partner lebenslangen Lernens“ verpflichtet war, zurück. In den Fachgesprächen dieses Bibliothekstages wünschten sich die Bibliothekare eine Arbeitsgruppe, in der sie in regelmäßigen Abständen die Praxis bibliothekspädagogischen Handelns diskutieren, die bisher gesammelten Erfahrungen auf dem Gebiet Benutzerschulung austauschen und neue Schulungskonzepte entwerfen könnten. Der Vorstand des Landesverbandes Thüringen im DBV griff diese Idee auf und bereitete die Gründung der AG Benutzerschulung vor. Entwicklungsziel war es, eine Arbeits- und Fortbildungsplattform für Bibliothekare, die sich im Bereich Benutzerschulung engagieren, zu schaffen, um den Diskurs zwischen den Thüringer

\footnotetext{
${ }^{11}$ Stichwort: Bibliothekar als Lernberater

${ }^{12}$ Vgl.: Schultka, Holger: Bibliothekspädagogik und die Arbeit der AG Benutzerschulung de Landesverbandes Thüringen im DBV. - In: Bibliothek : Forschung und Praxis. 29 (2005), Nr. 1, S. 59-65

${ }^{13}$ Homepage der AG: http://www.bibliothek.uni-erfurt.de/service/schul5.html

${ }^{14}$ Vgl.: 7. Thüringer Bibliothekstag in Schmalkalden am 27. Oktober 2001; DBV, Landesverband Thüringen im Deutschen Bibliotheksverband (Hrsg.): Bibliotheken - Partner lebenslangen Lernens. Erfurt 2001. Vgl. auch: Schultka, Holger: Thüringenweite AG Benutzerschulung gegründet. [Bericht der konstituierenden Sitzung]. http://www.bibliothek.uni-erfurt.de/service/texte/agbericht1.pdf. Erfurt 2002, S. 1 [Zugriff am 08.11.2004]
} 
Bibliotheken zum Thema „Pädagogische Arbeit in Bibliotheken“ über den Bibliothekstag hinaus dauerhaft zu etablieren.

Die konstituierende Sitzung der AG Benutzerschulung fand am 29. Mai 2002 in Weimar statt. Damit ist der Diskurs zum Thema Benutzerschulung und Bibliothekspädagogik zwischen den großen Thüringer Bibliotheken institutionalisiert worden. Zur Gründungssitzung hatte der damalige Fortbildungsbeauftragte des Landesverbandes Thüringen im DBV, Herr Dr. SimonRitz, eingeladen. Die großen wissenschaftlichen und öffentlichen Bibliotheken waren gebeten worden, einen interessierten Mitarbeiter zum ersten Treffen zu entsenden. Zur konstituierenden Sitzung waren ausschließlich Diplom-Bibliothekare gekommen.

In der AG arbeiten derzeit zwölf Diplom-Bibliothekare aus vier öffentlichen und acht wissenschaftlichen Bibliotheken ${ }^{15}$ zusammen. Die AG hat sich zum Ziel gesetzt, bibliothekspädagogische Fragen praxisnah zu diskutieren, bibliothekspädagogische Arbeitshilfen zu erarbeiten sowie Ideen zu den einzelnen Themenfeldern zusammenzutragen. Auch wollen die Mitglieder voneinander und miteinander lernen und sich so gegenseitig fortbilden. Die AG trifft sich zweimal im Jahr. Sie berichtet über ihre Arbeitsbesprechungen und veröffentlicht die Arbeitsergebnisse. ${ }^{16}$ Der Sprecher der AG hat die Aufgabe, die Arbeit zu koordinieren und zu den Arbeitsbesprechungen einzuladen.

\section{Zusammenarbeit zwischen öffentlichen und wissenschaftlichen Bibliotheken}

In der thüringenweiten AG Benutzerschulung arbeiten Bibliothekare aus öffentlichen und aus wissenschaftlichen Bibliotheken zusammen. Dies bringt Vor- und Nachteile, wobei die Vorteile überwiegen.

\section{$\underline{\text { Vorteile }}$}

Die Mitglieder der AG bringen bei den Arbeitstreffen unterschiedliche Bildungsstandpunkte und Lehrerfahrungen in die Diskussionen ein. Beim Nachdenken wird in der Regel auf die Bildungssituation in den Herkunftsbibliotheken rekurriert. Diese stellt die wichtigste Quelle für Bildungserfahrungen dar. Die Sichtweisen der ,öffentlichen’ und ,wissenschaftlichen' Bibliothekare befördern widerstreitende und dadurch konstruktive Diskussionen. Unterschiede bedingen Anregungen in beiderlei Richtung: Warum nicht auch in wissenschaftlichen Bibliotheken Infotexte vereinfachen, Fließtexte, wo es angebracht ist, in Übersichtstexte umwandeln oder Inhalte, wenn möglich, visualisieren und symbolisieren, was manchmal besser als eine Verbalisierung ist? Warum nicht in einer wissenschaftlichen Bibliothek einen literarischen Salon für Jugendliche veranstalten? Warum nicht Informationskompetenztrainings in öffentlichen Bibliotheken durchführen? Warum nicht das Seniorenkolleg der Universität in die öffentliche Bibliothek einladen?

Die Zusammenarbeit von Bibliothekaren aus öffentlichen und wissenschaftlichen Bibliotheken führt dazu, dass Klischees (wie z.B.: bibliothekspädagogische Veranstaltungen in wissenschaftlichen Bibliotheken sind immer ernst, in öffentlichen Bibliotheken hingegen edutainend und witzig) in Frage gestellt und ad absurdum geführt werden. Zudem wird das bibliotheks-

\footnotetext{
${ }^{15}$ In der AG vertretene Bibliotheken: öffentliche Bibliotheken: Stadt- und Regionalbibliothek Erfurt, Stadt- und Regionalbibliothek Gera, Ernst-Abbe-Bücherei Jena und Stadtbibliothek Rudolstadt; wissenschaftliche Bibliotheken: Bibliothek der Fachhochschule Erfurt, Universitäts- und Forschungsbibliothek Erfurt/Gotha, Universitätsbibliothek Ilmenau, Thüringer Universitäts- und Landesbibliothek Jena, Bibliothek der Fachhochschule Nordhausen, Bibliothek der Fachhochschule Schmalkalden, Bibliothek der Bauhaus-Universität Weimar und Herzogin Anna Amalia Bibliothek Weimar.

${ }^{16}$ Die Berichte und Arbeitsergebnisse bzw. die Hinweise auf die Arbeitsergebnisse können über die Homepage der AG (vgl. Fußnote 13) abgerufen werden.
} 
spartenübergreifende, curriculare Bildungsdenken befördert, sodass spartenübergreifende gestufte Bildungsprogramme möglich werden können. Es wird schnell erkannt, dass nicht wissenschaftliche Bibliotheken allein, insbesondere in der Form von Hochschulbibliotheken, Informationskompetenzen ausbilden, sondern dass sich ebenso öffentliche Bibliotheken mit ihren Veranstaltungen für Vorschüler und Schüler an der Entwicklung von Informationskompetenz beteiligen.

Alle grundlegenden pädagogischen Fragen (z. B. die: Wie zergliedert man eine Veranstaltung in Lernphasen oder Aktionsphasen oder Sozialphasen oder Inhaltsphasen?) können stets gemeinsam diskutiert werden.

\section{$\underline{\text { Nachteile }}$}

Manche bibliothekspädagogischen Aktivitäten oder Veranstaltungsarten, sind Bibliothekssparte- bzw. -typ-spezifisch. Zum Beispiel: Informationskompetenzveranstaltungen im Rahmen der Propädeutik gibt es in der Regel nur an Hochschulbibliotheken. Bibliothekspädagogische Veranstaltungen für Kindergartenkinder werden nur von öffentlichen Bibliotheken durchgeführt.

Werden in einer AG-Sitzung Fragen zu solchen Bildungsspezifika aufgeworfen, kann es passieren, dass die Arbeit an einem solchen Thema eingestellt oder das Thema verändert werden muss, weil sich nicht alle AG-Mitglieder für den Gesprächsgegenstand interessieren oder sie keine Übertragungen auf ihre Arbeit vornehmen können, somit keine Anregungen für ihre Arbeit durch die Diskussion erhalten würden.

\section{$\underline{\text { Arbeitsergebnisse }}$}

Die AG hat ein Positionspapier „Bibliothekspädagogik“17 erarbeitet (verabschiedet am 18. September 2003), in dem Standpunkte zur edukativen Arbeit in Bibliotheken formuliert worden sind.

Einige Treffen der AG waren vollständig, einige teilweise der Fortbildung gewidmet. Fortbildungsthemen waren:

- Elemente der didaktischen Situation,

- „Kompetent Informationskompetenz formen: Wie gestalte ich nutzerorientierte Schulungen?“ (Referentin: Katrin Richter),

- $\quad$ Lehrmittel $^{18}$ zur Entwicklung von Informationskompetenz, welche man in Veranstaltungen für Schüler und/oder Studierende einsetzen kann.

Die AG hat die Broschüre „Unterricht + Bibliothek“ ${ }^{19}$ in enger Zusammenarbeit mit dem ThILLM (Thüringer Institut für Lehrerfortbildung, Lehrplanentwicklung und Medien) erarbeitet. Die Broschüre enthält u. a. eine Aufgabensammlung mit Lösungsvorschlägen. Zudem

\footnotetext{
${ }^{17}$ AG Benutzerschulung des Landesverbandes Thüringen im DBV: Bibliothekspädagogik - ein Positionspapier. In: Bibliotheksdienst 2 (2004) S. 161-164. - Auch von der Homepage der AG (vgl. Fußnote 13) aus abrufbar. http://www.bibliothek.uni-erfurt.de/service/texte/positionspapier.pdf

${ }^{18}$ Die Liste der Hilfsmittel (systematisch und alphabetisch geordnet): http://www.bibliothek.unierfurt.de/service/texte/hilfsmittel.pdf. Einige der Hilfsmittel sind ausführlich besprochen in: Schultka, Holger: Bibliothekspädagogik - Schwerpunkt Informationskompetenz. In: Bibliotheksdienst 9 (2004) S. 1107-1119 und 10 (2004) S. 1301 - 1315

${ }^{19}$ ThILLM; Schenk, Renate (Red.); Schultka, Holger (Red.): Unterricht + Bibliothek. Bad Berka 2004 (Materialien; 94). - Mit 1 CD-ROM-Beil.
} 
hat die AG die Erfurt-Gothaer Seminarfach-Initiative ${ }^{20}$ unterstützt.

\section{Die Erfurt-Gothaer Seminarfach-Initiative}

Die Stadt- und Regionalbibliothek Erfurt, die Stadtbibliothek Gotha, die Fachhochschulbibliothek Erfurt und die Universitäts- und Forschungsbibliothek Erfurt/Gotha haben gemeinsam eine Broschüre herausgegeben, in der den Seminarfachlehrern das edukative Angebot der Bibliotheken vorgestellt wird. Ziel der Broschüre war es, den Run auf die Universitätsbibliothek Erfurt zu bremsen und die Gymnasialklassen weitgehend gleichmäßig auf alle großen Bibliotheken der Region Erfurt-Gotha zu verteilen.

Das Angebot der Erfurt-Gothaer Seminarfach-Initiative sieht wie folgt aus:

\begin{tabular}{|l|c|c|c|c|c|c|}
\hline Angebot & \multicolumn{5}{|c|}{ Wer bietet was? } \\
\hline & \multicolumn{5}{|c|}{ Bibliothek } \\
\hline Ein Rundgang durch die Bibliothek & 1 & 2 & 3 & 4 & 5 \\
\hline Thematische Einführung & + & + & + & + & + \\
\hline Recherchetraining & + & + & & & \\
\hline Literaturlisten erstellen + Zitierregeln & + & + & & + & \\
\hline Bücherkiste & & & & + & \\
\hline Selbst in der Bibliothek unterrichten & + & & & & \\
\hline Vorstellen der Seminarfacharbeiten in der Bibliothek & + & & & + & + \\
\hline Die Angebote miteinander kombinieren & + & + & & & \\
\hline Weitere Angebote & + & + & & + & + \\
\hline
\end{tabular}

Legende zur Tabelle:

1 = Stadt- und Regionalbibliothek Erfurt

2 = Stadtbibliothek „Heinrich Heine“ Gotha

3 = Bibliothek der Fachhochschule Erfurt

4 = Universitäts- und Forschungsbibliothek Erfurt/Gotha, Universitätsbibliothek Erfurt

5 = Universitäts- und Forschungsbibliothek Erfurt/Gotha, Forschungsbibliothek Gotha

Die Broschüre der Erfurt-Gothaer Initiative „Seminarfach - Unterricht in der Bibliothek“ ist in den beteiligten Bibliotheken ausgelegt und an die Gymnasien in Erfurt und Gotha sowie des Umlandes verschickt worden. Seitdem verteilt sich die edukative Arbeit auf alle beteiligten Bibliotheken weitgehend gleichmäßig. Die Resonanz auf das unterbreitete Angebot ist bis jetzt ungebrochen gut.

\section{Ausblick}

Bibliothekspädagogisches Handeln ist ein offenes Konzept. Es gibt keinen Endpunkt, sondern stets nur neue Herausforderungen und Zielstellungen und den Optimismus, ein Ideal erreichen zu können. Mit sich wandelnden Rahmenbedingungen für die Bildungsarbeit in Bibliotheken wird man leben müssen. Eine Besonderheit der Arbeit besteht darin, dass oft nur für eine begrenzte Zeit mit den Lerngruppen zusammengearbeitet werden kann (meist nur 90 Minuten lang). Desiderate für die Arbeit sind:

- Ganzheitliche Konzepte für die Bildungsarbeit in Bibliotheken und die Gestaltung der Bibliothek als Lernort. (Derzeit werden meist nur Bildungsansätze und Teilaspekte realisiert.)

\footnotetext{
${ }^{20}$ Stadt- und Regionalbibliothek Erfurt [u.a.]: Seminarfach - Unterricht in der Bibliothek. Stand: 24.02.2003. Erfurt 2003. http://www.bibliothek.uni-erfurt.de/service/texte/seminarfachunterricht.pdf
} 
- Bibliothekspädagogische Lehrmittel. (Einige Lehrmittel gibt es inzwischen, doch reicht die Anzahl an Hilfsmitteln bei weitem noch nicht aus.)

- $\quad$ Eine fundierte bibliothekspädagogische Theorie und ein praxisorientiertes Lehrwerk. - Bibliothekspädagogik als Lehrinhalt in den bibliothekarischen Studiengängen.

Ich wünsche mir für die Zukunft, dass die pädagogische Arbeit in allen Bibliotheken phantasiereicher und vielfältiger wird und dass Bibliotheken bibliothekspädagogische Abteilungen gründen, zumindest einen Mitarbeiter bestimmen, der sich auf die pädagogische Arbeit spezialisieren kann. Ich wünsche mir, dass Hochschulbibliotheken „Informationskompetenz“ nur als einen Lehrinhalt unter weiteren realisieren wollen und dass wissenschaftliche und öffentliche Bibliotheken untereinander Bildungskooperationen eingehen. Ebenso wünsche ich mir, dass in Bibliotheken nicht nur ein einziges pädagogisches Handlungsmodell präferiert wird, sondern dass mehrere eine Chance erhalten. (Jede Bibliothek muss selbst schauen, welche pädagogischen Angebote sie mit ihren jeweiligen Ressourcen realisieren kann.) 